\title{
Reviewers 2010
}

(C) Springer-Verlag 2010

In addition to the assistance of the Editorial Board, the Editors and the Journal would also like to thank the following referees for their valuable input during 2010:
Agostini, F.

Anglin, R,

Arfken, C.

Austin, M.P.

Battle, C.

Beck, C.

Ben Dor, R.

Biltsza, J,

Boyce, P.

Boylan, K.

Brandon, A.

Brotea, A.

Carter, D.

Carvalhal, A.

Castle, D.

Chason-Tabor, L.

Chatterji, S.

Chaaya, M.

Chaudron, L.

Collins, S.

Condon, J.

Conroy, S.

Cox, J.L.

Dalfen, A.

Deligiannidis, K.
Diaz-Granados, N.

Einarson, A.

El-Hage, W.

Elhayani, A.

Eriksson, E.

Ezechi, O.

Fairbrother, N.

Faisal-Cury, A.

Fink, N.

Flynn, H.

Freeman, E.

Freeman, M.

Frey, B.

Glangeaud-Freudenthal, N.

Green, $\mathrm{S}$.

Grigoriadis, S.

Haskett, R.

Heaphy, E.

Hofecker Fallahpour, M.

Jadresic, E.

Joffe, $\mathrm{H}$.

Kammerer, M.

Kim-Godwin, Y.S.

Kimerling, R.

Koren, G.
Kornstein, S.

Kuan, A.

Lack, C.

Lancaster, C.

Lee, $\mathrm{K}$.

Leventhal, J.

Levitan, R.

Lipman, E.

Lislelott, A.

Lord, C.

Macdougall, M.

Matthey, S.

McKinnon, M.

Meltzer Brody, S.

Meinlschmidt,G.

Newport, J.

O'Brien, P.M.S.

O'Hara, M.

Paris, R.

Pauleta, J.

Pearlstein, T

Peer, M.

Poudevigne, M.

Rapkin, A.

Reck, C.
Reid, R.

Resnick, H.

Restifo, K.

Robinson, G.E.

Ross, L.

Rowa, K

Rowe, $\mathrm{H}$.

Sassi, R.

Schmidt, P.

Sharma, V.

Shea, A.

Sit, D.

Smith, M.

Soreni, N.

Spinelli, M.

Stotland, N.

Stowe, Z.

Suchman, N.

Talbot, N.

Tandon, S. D.

Van lieshout, R.

Videbech, P.

Vigod, S.

Vythilingum, B.

Wadwha, P. 